\title{
Mesalazine Has No Effect on Mucosal Immune Biomarkers in Patients with Diarrhea-Dominant Irritable Bowel Syndrome Referred to Shariati Hospital: A Randomized Double-Blind, Placebo-Controlled Trial
}

Mohammad Reza Ghadir ${ }^{1}$, Mehri Poradineh ${ }^{2}$, Masoud Sotodeh ${ }^{3}$, Reza Ansari ${ }^{4}$, Shadi Kolahdoozan ${ }^{2}$, Ahmad Hormati ${ }^{5}$,Mohammad Hosein Yousefi ${ }^{6}$,Samaneh Mirzaei ${ }^{2}$, Homayoon Vahedi ${ }^{4 *}$

\section{ABSTRACT}

1. Associate Professor, Qom Gastroenterology and Hepatology Research Center, ShahidBeheshti Hospital, Qom University of Medical Sciences, Qom, Iran

2. Researcher, Digestive Disease Research Institute, Shariati Hospital, Tehran University of Medical Sciences, Tehran, Iran

3. Professor, Digestive Disease Research Institute, Shariati Hospital, Tehran University of Medical Sciences, Tehran, Iran

4. Associate Professor, Digestive Disease Research Institute, Shariati Hospital, Tehran University of Medical Sciences, Tehran, Iran

5. Assistant Professor, Qom Gastroenterology and Hepatology Research Center, ShahidBeheshti Hospital, Qom University of Medical Sciences, Qom, Iran

6. Researcher and Bio-statistics Expert, Qom Gastroenterology and Hepatology Research Center, Shahid Beheshti Hospital, Qom University of Medical Sciences, Qom, Iran

\footnotetext{
* Corresponding Author:

Homayoon Vahedi, MD

Digestive Disease Research Center, Shariati Hospital, Tehran University of Medical Sciences, Tehran, Iran Telefax: + 982182415400

Email: vahedi@ams.ac.ir
}

Received: 28 Jul. 2016

Accepted: 11 Nov. 2016

\section{BACKGROUND}

Intestinal mast cells may cause gastrointestinal symptoms in patients with diarrhea-dominant irritable bowel syndrome (IBS). The objective of this study was to determine the effect of mesalazine on the number of lamina propria mast cells and clinical manifestations of patients with diarrhea-dominant IBS referred to Shariati Hospital affiliated to Tehran University of Medical Sciences.

\section{METHODS}

This was a randomized placebo-controlled double-blind trial conducted on 49 patients with diarrhea-dominant IBS. The patients were randomly assigned to one of the experiment or control groups. The patients in experiment group took 2400 $\mathrm{mg}$ mesalazine daily in three divided doses for 8 weeks and the patient in control group took placebo on the same basis. Our first targeted outcome was an assigned downturn of mast cells number to the safe colonic baseline and the next one was a marked palliation of disease symptoms. Data were analyzed conforming intentionto-treat method. We used MANCOVA test to compare our both assigned outcomes in the two groups. We also compared the data with baseline values in both groups. All statistical tests were performed at the significance level of 0.05 .

\section{RESULTS}

There was no significant difference between Mesalazine and placebo groups regarding the number of mast cells ( $p$ value $=0.396)$, abdominal pain $(p$ value $=0.054)$, bloating $(p$ value $=0.365)$, defecation urgency $(p$ value $=0.212)$, and defecation frequency $(p$ value $=0.702)$.

\section{CONCLUSION}

Mesalazine had no significant effect either on the number of mast cells or on the severity of disease symptoms. This finding seems to be inconsistent with the hypothesis indicating immune mechanisms as potential therapeutic targets in IBS.

The possible difference in this effect of Mesalazine should be evaluated in further studies among populations varying in race, ethnic, and geographical characteristics.

KEYWORDS: Mesalazine, IBS-D, Symptoms, Intestinal Mucosa, Mast cells, Biomarkers

Please cite this paper as:

Ghadir MR, Poradineh M, Sotodeh M, Ansari R, Kolahdoozan S, Hormati A, Yousefi MH,Mirzaei S, Vahedi H. Mesalazine Has No Effect on Mucosal Immune Biomarkers in Patients with Diarrhea-Dominant Irritable Bowel Syndrome Referred to Shariati Hospital: A Randomized Double-Blind, Placebo-Controlled Trial. Middle East J Dig Dis 2017;9:20-25. DOI: 10.15171/mejdd.2016.47

\section{INTRODUCTION}

Irritable bowel syndrome (IBS) is a functional gastrointestinal disorder characterized by recurrent abdominal pain, discomfort, and bloating along with annoy- 
ing changes in bowel habit. None of the theories suggesting a single organic cause for the disease is fully plausible and most current theories ascribe it as a multifactorial disease. ${ }^{1-3}$

Some known factors including stress, altered gut microbiome, gastroenteritis, bile and short-chain fatty acids, which provoke serotonin release, may contribute to IBS symptoms via intensification of both colonic motility and permeability. ${ }^{4-6}$ IBS affects up to $20 \%$ of population in developed countries and accused for up to half of overall referrals to gastroenterologists in addition to a remarkable proportion of visits to primary care physicians. About one third of IBS cases are diarrhea-predominant or IBS-D. ${ }^{7-9}$

For several decades, we have had to prescribe drugs just for some sort of symptoms relief among patients with IBS. This shortcoming necessitates extensive studies to develop curative treatments for the disease. However, our inadequate knowledge about the disease pathophysiology made it hard to achieve. On the other hand, clinical research about newly developed drugs always improved our insight into the pathophysiological aspects of many diseases. Accordingly, we must evaluate Mesalazine effects on IBS symptoms and immunological mechanisms, which may contribute to those symptoms. Since the treatment of IBS-D has not been satisfying, we decided to determine the potential benefits of Mesalazine in pacifying its symptoms as well as restriction of intestinal mucosal mast cells aggregation in a randomized, controlled trial.

Some studies have shown an elevated number of immune cells especially mast cells in different segments of gastrointestinal tract. ${ }^{9-11}$ Increased number of mast cells has been observed in the terminal ileum, jejunum, and entire colon among patients with IBS. ${ }^{10}$ In a study carried out in Ireland, mast cells increased significantly in the caecum of patients with IBS. Rectal sensitivity was increased in patients with IBS-D with moderately increased mucosal mast cells. ${ }^{11}$ Philpott, Gibson, and Thienhave shown an association between the intensity of mast cells mucosal aggregation and severity of abdominal pain, depression, and anxiety. ${ }^{12}$ Although it is generally accepted that finding an objective immunological target can present a particular etiology as a contributing factor, there is not decisive evidence for the usefulness of anti-inflammatory drugs prescription to patients with
IBS. ${ }^{13}$ Mesalazine (5-aminosalicylic acid) is a known anti-inflammatory drug, which is commonly applied for the treatment of chronic inflammatory bowel diseases such as ulcerative colitis and Crohn's disease. ${ }^{14}$ Mesalazine probably activate nuclear receptors such as the gamma form of proxisome proliferation activating receptors, which downregulate inflammatory process and decrease inflammatory cytokines release. ${ }^{15}$ Moreover, mesalazine that does not have antibiotic activity compared with sulfasalazine, may also act by downregulating mast cells function, a major feature in IBS immunopathology. This downregulation occurs in both human and rodents. ${ }^{16,17}$

\section{MATERIALS AND METHODS}

It was a double blind randomized controlled trial with initial samples of 74 patients. During the 2-year period of the study, we screened 87 outpatients with IBS-D aged more than 18 years in accordance to clinical criteria of Rome III. We used sequential sampling method. Our inclusion criteria were having IBS-D and age more than 18 years. Exclusion criteria were: pregnancy, breast feeding, improper use of contraceptives, substantial abdominal surgery, major allergic diseases such as asthma in patients or their first degree relatives, celiac disease, history of any organic or psychiatric disorders, and finally the use of anti-inflammatory drugs, antibiotics, and mast cell stabilizers.

Four patients did not meet our inclusion or exclusion criteria and excluded after initial screening. During the process of obtaining informed consents, nine patients denied signing papers and 74 patients remained in our study.

We assigned our cases equally and randomly to one of the experiment $(800 \mathrm{mg}$ Mesalazine three times a day after meal for 8 weeks) or control (placebo on the same basis) groups. We performed case allocation by block randomization method with block size of four. After creating experiment and placebo groups, 17 cases from the control group and 8 cases from the experiment group dropped out due to failure to return to the first follow-up session. Then we had to continue with 49 patients, 29 in experiment group, and 20 in control group.

For the purpose of blinding, we ordered the same capsules of Mesalazine and placebo in same bottles and packages. True content of each bottle could only be determined by matching its serial number with the same 
Table 1: Demographic details and baseline characteristics of the patients

\begin{tabular}{lccc}
\hline Variables & Control (20) & Experiment (29) & $\boldsymbol{p}$ value \\
\hline Age: Mean, (S.D*) & $32.1(7.9)$ & $34.5(8.8)$ & 0.312 \\
\hline Male/female & $13 / 7$ & $21 / 8$ & 0.591 \\
\hline Gastrointestinal symptoms: N (\%) & $17(85.0 \%)$ & $25(86.2 \%)$ & 0.902 \\
\hline Abdominal pain: N (\%) & $16(80.0 \%)$ & $20(69.0 \%)$ & 0.335 \\
\hline Incomplete defecation: N (\%) & $11(55.0 \%)$ & $20(69.0 \%)$ & 0.263 \\
\hline Abdominal urgency: N (\%) & $16(80.0 \%)$ & $23(79.3 \%)$ & 1.0 \\
\hline IHC count in ascending colon: Mean, (S.D) & $104.6(60)$ & $81.4(54.1)$ & 0.245 \\
\hline IHC** count in descending colon: Mean (S.D) & $107(72.3)$ & $87.1(54.9)$ & 0.329 \\
\hline IBS symptoms, Mean \pm S.E.M*** & & $4.7 \pm 0.6$ & 0.074 \\
\hline Abdominal pain severity & $3.2 \pm 0.6$ & $4.8 \pm 0.6$ & 0.466 \\
\hline Abdominal bloating severity & $4.2 \pm 0.6$ & $4.1 \pm 0.7$ & 0.352 \\
\hline Defecation urgency severity & $3.1 \pm 0.8$ & $3.8 \pm 0.6$ & 0.298 \\
\hline Daily defecation frequency & $3.0 \pm 0.4$ & & \\
\hline
\end{tabular}

* Standard Deviation, ** Immunohistochemistry, ${ }^{* * *}$ Standard Error of the Mean

Table 2: Response of gastrointestinal symptoms to Mesalazine among the studied patients

\begin{tabular}{lccc}
\hline Variables & Control (20) & Experiment (29) & $p$ value \\
\hline Gastrointestinal symptoms: N (\%) & & & 0.401 \\
\hline Abdominal pain & $10(50.0 \%)$ & $18(62.1 \%)$ & 0.378 \\
\hline Incomplete defecation & $13(65.5 \%)$ & $15(51.7 \%)$ & 0.235 \\
\hline Abdominal urgency & $7(35.0 \%)$ & $15(51.7 \%)$ & 0.187 \\
\hline Abdominal bloating & $15(75.0)$ & $17(58.6 \%)$ & 0.054 \\
\hline IBS symptoms: Mean \pm S.E.M* & & & 0.365 \\
\hline Abdominal pain severity & $1.0 \pm 0.3$ & $2.1 \pm 0.4$ & 0.212 \\
\hline Abdominal bloating severity & $1.9 \pm 0.5$ & $2.5 \pm 0.5$ & 0.702 \\
\hline Defecation urgency severity & $1.0 \pm 0.4$ & $1.8 \pm 0.4$ & $1.9 \pm 0.3$ \\
\hline Defecation frequency per day & $2.1 \pm 0.3$ & & \\
\hline
\end{tabular}

* Standard Error of the Mean

Table 3: Effect of Mesalazine treatment on mucosal immune cells

\begin{tabular}{|c|c|c|c|c|c|}
\hline \multirow{2}{*}{ Variable } & \multicolumn{2}{|c|}{ Placebo (20) } & \multicolumn{2}{|c|}{ Mesalazine (29) } & \multirow{2}{*}{$p$ value } \\
\hline & Baseline & Final & Baseline & Final & \\
\hline $\mathrm{IHC}^{*}$ count in descending colon: Mean \pm S.E.M $\mathrm{M}^{* *}$ & $109.2 \pm 16.8$ & $81.6 \pm 12.4$ & $87.1 \pm 10.2$ & $89.2 \pm 12.4$ & 0.396 \\
\hline
\end{tabular}

* Immunohistochemistry, ** Standard Error of the Mean

numbers on our computer database that was not accessible by researchers and other people involved in assigning or following the patients until the end of data analysis.

Collected data from the patients including age, sex, and IBS symptoms and their severity (before and after 8-week period) entered to their questionnaires. The data gathered from the first and second times colonoscopy (before and after 8-week period) along with microscopic data created by pathological examinations of large intestinal mucosal tissues obtained from two times biopsy performed during each session of colonoscopy entered to the questionnaires too. To define the severity of symptoms, we used visual 
analogous scales (VAS) by asking the patients to define the severity of a specific symptom by a number ranging from zero (no symptom) to 100 (maximum severity of symptom). For microscopic examination of the mucosal tissues of colonic wall, we took two biopsy samples in both first and second sessions of colonoscopy. In the first time colonoscopy, we took two biopsy samples from the proximal and descending colon of each patient. During the second session, we took two biopsy samples from descending colon. We referred the biopsy samples for routine $H \& E$ staining histological examination as well as immunohistochemistry. We used polyclonal antibody directed against CD117 (mast cells marker) to characterize mucosal immune cells. We were able to count immune cells by a microscope, using either H\&E staining method or immunohistochemically stained sections that can define even the subtypes of immune cells. The benefit of this method is to adapt a morphometric point counting procedure to determine the area covered by different types of cells in the lamina properia. Results show the number of mast cells per field. We believe this method can minimize the measuring bias and maximize counting accuracy.

We applied intention-to-treat (ITT) analysis. Hence, the data of all randomly assigned patients who had received at least one dose of Mesalazine or placebo were considered for analysis. We used the principle of last observation carried forward (LOCF) for estimating missing data. We assessed a baseline for evaluating the demographic and primary efficacy data homogeneity using t test for continuous covariates such as age and total number of inflammatory mucosal cells. We also assessed the assumptions of normality and variance homogeneity for the efficacy variables at the end of treatment. We performed an analysis of covariance (ANCOVA), adjusted for the baseline values to compare the primary and secondary variables between the treatment groups at the end of the 8-week period. All the safety variables were analyzed on the safety population. We performed all statistical analysis at a significance level of 0.05 using SPSS software version 21.

The registration number of this study at the Iranian Registry of Clinical Trials is RCT2012072910230N2. We got ethical approval from the ethics committees of DDRI (Digestive Disease Research Institute) and Tehran University of Medical Sciences (TUMS) Immunological studies on mucosal biopsies. Committee of Digestive Diseases
Research Institute of TUMS has approved the study too.

\section{RESULTS}

Demographic details and baseline characteristics of the studied patients are shown in table1.

Table 2 displays the response of gastrointestinal symptoms to Mesalazine among the patients. As we displayed in this table, Mesalazine had no significant effect on abdominal pain ( $p=0.054)$, bloating ( $p=0.365)$, defecation urgency $(p=0.212)$, or defecation frequency $(p=0.702)$ in patients with IBS-D.

Table 3 shows that there was no significant difference between Mesalazine and placebo on the total mucosal immune cell counts of the patients with IBS-D ( $p=0.396)$.

\section{DISCUSSION}

During the 2-year period of our study, we screened 87 patients with IBS-D but only 49 patients remained in our study. Of the remaining patients, 29 patients (59\%) entered to 2-month period of our intervention with Mesalazine and 20 patients (41\%) took placebo for a similar period. As we showed, Mesalazine did not have any effect either on disease symptoms or on the mast cells aggregation in lamina propriain patients with IBS-D compared with placebo.

In a study in Italy in 2009, Corinaldesi concluded that Mesalazine decreased the number of mast cell (Variables $=0.0014$ ) and improved general wellbeing ( $p=0.038)$. However, it did not have any significant effect on IBS symptoms. The difference between the results of these two studies could be due to different patients (all form of IBS instead of IBS-D only) and different geographical area. ${ }^{18}$ In some patients with IBS, a lowgrade inflammation that is rich in mucosal mast cells, can affect both small and large intestine. ${ }^{10,12,17}$ Moreover, serological markers of this lowgrade inflammation have drawn attention of researchers to ascribe IBS as an inflammatory disease. ${ }^{18-19}$

The most recent theories suggest that mast cells can play a key role in the regulation of gastrointestinal visceral sensitivity and vascular permeability. There are several studies that reported elevated count of mast cells in the intestinal mucosa of patients with IBS. ${ }^{10,20,21} \mathrm{Be}-$ sides; at least one study reported the increased numbers of mast cells in the terminal ileum, ascending colon, and 
rectum of patients with IBS-D.11

Our study revealed that, Mesalazine had no significant effect on abdominal pain ( $p=0.054)$, bloating ( $p=0.4$ ), and defecation urgency $(p=0.2)$ in patients with IBS-D. These finding are similar to the results of many previous studies. ${ }^{12,18,20,22}$ However, our finding, which denies the effect of Mesalazine on mucosal mast cells count in patients with IBS-D, is against the results of similar studies among patients with all forms of IBS.

Role of colonic mast cell infiltration and mediator release in proximity to mucosal innervations in the perception of abdominal pain in patients with IBS cannot be ignored. ${ }^{22}$ In a multicenter randomized controlled trial with sample size of 108 patients, Leighton and his colleagues suggested that two grams oral Mesalazine twice a day for 12 weeks could be beneficial for patients with IBS-D especially in decreasing mast cells. ${ }^{23}$ However, in our study there was not a statistically significant decrease in the number of mast cells in the treatment group compared with the placebo group. Our small sample size as well as potential different effect of Mesalazine in different populations could be the possible explanations for those controversial findings.

Our small sample size was one of our limitations. Because of psychosocial disturbances in patients, it was not possible for us to recruit more participants. Moreover, it was not possible for us to ensure that our participants consumed their drugs or did not. Indeed, we are not aware from the compliance of our patients.

In conclusion, our data suggest that mesalazine is not an efficient drug in pacifying symptoms in IBS-D. In our study, neither intensity nor duration of pain was significantly reduced by mesalazine. Further research in this field with larger sample size is recommended.

\section{ACKNOWLEDGMENT}

We would like to express our sincere thanks to Dr Mohamadreza Aghamirsalim and Dr Mani Beigi whose constructive comments enabled us to perform this trial much better.

\section{Source of funding:}

Digestive Disease Research Institute, Shariati Hospital, Tehran University of Medical Sciences, Teh- ran, Iran

(RegistrationIDinIRCT.IR:IRCT2015083117719N2)

\section{CONFLICT OF INTEREST}

The authors declare no conflict of interest related to this work.

\section{REFERENCES}

1. Talley NJ, Zinsmeister AR, Van Dyke C, Melton LJ 3rd. Epidemiology of colonic symptoms and the irritable bowel syndrome. Gastroenterology 1991;101:927-34.

2. Drossman DA, Li Z, Andruzzi E, Temple RD, Talley NJ, Thompson WG, et al. U.S. householder survey of functional gastrointestinal disorders. Prevalence, sociodemography, and health impact. Dig Dis Sci 1993;38:1569-80. doi:10.1007/BF01303162

3. American College of Gastroenterology Task Force on Irritable Bowel Syndrome, Brandt LJ, Chey WD, FoxxOrenstein AE, Schiller LR, Schoenfeld PS, et al.An evidence-based position statement on the management of irritable bowel syndrome.Am J Gastroenterol 2009;104Suppl 1:S1-35. doi: 10.1038/ajg.2008.122.

4. Hahn BA, Saunders WB, Maier WC. Differences between individuals with self-reported irritable bowel syndrome (IBS) and IBS-like symptoms. Dig Dis Sci 1997;42:2585-90. doi : 10.1016/S0016-5085(03)82564-7

5. Saito YA, Locke GR, Talley NJ, Zinsmeister AR, Fett SL, Melton LJ 3rd. A comparison of the Rome and Manning criteria for case identification in epidemiological investigations of irritable bowel syndrome. Am J Gastroenterol 2000;95:2816-24. doi:10.1016/S0016-5085(98)83380-5

6. Thompson WG, Irvine EJ, Pare P, Ferrazzi S, Rance L. Functional gastrointestinal disorders in Canada: First population-based survey using Rome II criteria with suggestions for improving the questionnaire. Dig Dis Sci 2002;47:225-35. doi:10.1023/A:1013208713670

7. Everhart JE, Renault PF. Irritable bowel syndrome in office-based practice in the United States. Gastroenterology 1991;100:998-1005.

8. Schuster MM. Diagnostic evaluation of the irritable bowel syndrome. Gastroenterol Clin North Am 1991;20:269-78.

9. Guilarte M, Santos J, de Torres I, Alonso C, Vicario M, Ramos L et al. Diarrhea-predominant IBS patients show mast cell activation and hyperplasia in the jejunum. Gut 2007;56:203-9. doi:10.1136/gut.2006.100594

10. Barbara G, Stanghellini V, De Giorgio R, Cremon C, Cottrell GS, Santini D, et al. Activated mast cells in proximity to colonic nerves correlate with abdominal pain in irritable bowel syndrome. Gastroenterology 2004;126:693-702. doi:10.1053/j.gastro.2003.11.055

11. Park JH, Rhee PL, Kim HS, Lee JH, Kim YH, Kim JJ, et al. Mucosal mast cell counts correlate with visceral hypersensitivity in patients with diarrhea predominant irritable 
bowel syndrome.J Gastroenterol Hepatol 2006;21:71-8. doi: 10.1111/j.1440-1746.2005.04143.x

12. Hamish Philpott, Peter Gibson, Frank Thien. Irritable bowel syndrome - An inflammatory disease involving mast cells.Asia Pac Allergy 2011;1:36-42. doi: 10.5415/ apallergy.2011.1.1.36.

13. Andrews CN, Petcu R, Griffiths T, Bjarnason J, Chapman K, Cellars L, et al. Mesalazine alters colonic mucosal proteolytic activity and fecal bacterial profiles in diarrhea-predominant irritable bowel syndrome (IBSD) Gastroenterology 2008;134:A548-A. doi:10.1016/ S0016-5085(08)62558-5

14. Baumgart DC, Sandborn WJ. Inflammatory bowel disease: clinical aspects and established and evolving therapies. Lancet 2007;369:1641-57. doi:10.1016/S01406736(07)60751-X

15. Rousseaux C, Lefebvre B, Dubuquoy L, Lefebvre P, Romano $\mathrm{O}$, Auwerx $\mathrm{J}$, et al. Intestinal anti-inflammatory effect of 5-aminosalicylic acid is dependent on peroxisome proliferator-activated receptor- gamma. J Exp Med 2005;201:1205-15. doi: 10.1084/jem.20041948

16. Fox CC, Moore WC, Lichtenstein LM. Modulation of mediator release from human intestinal mast cells by sulfasalazine and 5-aminosalicylic acid. Dig Dis Sci 1991;36:179-84. doi:10.1007/BF01300753

17. Bissonnette EY, Enciso JA, Befus AD. Inhibitory effects of sulfasalazine and its metabolites on histamine release and TNF-alpha production by mast cells. $J \mathrm{Im}$ munol 1996;156:218-23. doi: 10.1111/j.1365-2249.1995. tb06639.x

18. David B. Ramsay, Sindu Stephen, Marie Borum, LysandraVoltaggio, David B. Doman. Mast Cells in Gastrointestinal Disease. Gastroenterol Hepatol (NY) 2010;6:7727.

19. Leighton MP, Lam C, Mehta S, Spiller RC. Efficacy and mode of action of Mesalazine in the treatment of diarrhea-predominant irritable bowel syndrome (IBS-D): study protocol for a randomized controlled trial. Trials 2013 9;14:10. doi :10.1186/1745-6215-14-10

20. Mayer EA. Clinical practice. Irritable bowel syndrome. $N$ Engl J Med 2008;358:1692-9. doi: 10.1056/NEJMcp0801447

21. R. Corinaldesi, V. Stanghellini, C. cremon, L. Gargano, R. F. Cogliandro, R. De Giorgio, et al: Effect of Mesalazine on mucosal immune biomarkers in irritable bowel syndrome: a randomized controlled proof-of-concept study. Aliment Pharmacol Ther 2009;30:245-52. doi:10.1111/ j.1365-2036.2009.04041.x

22. Barbara G, De Giorgio R, StanghelliniV,Cremon C, Salvioli B, Corinaldesi R. New pathophysiological mechanisms in irritable bowel syndrome. Aliment Pharmacol Ther 2004;20:1-9. doi: 10.1111/j.13652036.2004.02036.x

23. Bengtsson M, Ohlsson B, Ulander K. Development and psychometric testing of the Visual Analogue Scale for Ir- ritable Bowel Syndrome (VAS-IBS). BMC Gastroenterol 2007;7:16. doi: 10.1186/1471-230X-7-16 